\title{
Gas chromatography-mass spectrometry and high-performance liquid chromotagraphy analysis of the drug absorption characteristics in the buccal mucosa via a circulating device
}

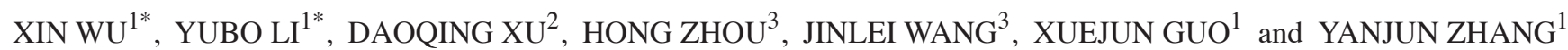 \\ ${ }^{1}$ School of Traditional Chinese Materia Medica, Tianjin University of Traditional Chinese Medicine; \\ ${ }^{2}$ Tianjin Zhongxin Pharmaceutical Group Corporation Limited, Tianjin 300193; ${ }^{3}$ No.6 Pharmaceutical Factory \\ of Tianjin Zhongxin Pharmaceutical Co., Qingguang North, Tianjin 300401, P.R. China
}

Received September 25, 2014; Accepted October 15, 2014

DOI: $10.3892 /$ br. 2014.382

\begin{abstract}
The aim of the present study was to investigate the characteristics of Su Xiao Jiu Xin dripping pill absorption in the buccal mucosa of healthy volunteers. This pill is a traditional Chinese medicine that is widely used as an emergency treatment for cardiovascular and cerebrovascular diseases. It is sublingually administered and can be absorbed in the buccal mucosa. In the present study, a method was developed to investigate the absorption characteristics in the buccal mucosa of healthy volunteers via a circulating device by gas chromatography-mass spectrometry and high-performance liquid chromatography. The five main efficacy components associated with cardiovascular and cerebrovascular diseases, which are borneol, isoborneol, ligustilide, $n$-butylphthalide and ferulic acid, were detected and rapidly absorbed. Among these components, four exhibited good absorption, thus confirming that the method developed is efficient for analysis of the absoption characteristics.
\end{abstract}

\section{Introduction}

$\mathrm{Su}$ Xiao Jiu Xin dripping pill (SXJXDP) is a traditional Chinese medicine (TCM), which is widely used to treat cardiovascular and cerebrovascular diseases. SXJXDP is composed of Chuanxiong (Rhizoma Chuanxiong) and Bing pian (Borneolum) (1). Phytochemical studies have shown that SXJXDP contains numerous types of compounds, such as borneol and isoborneol from Borneolum, lactones (ligustilide

Correspondence to: Professor Yanjun Zhang, School of Traditional Chinese Materia Medica, Tianjin University of Traditional Chinese Medicine, 312 Anshan West Road, Tianjin 300193, P.R. China

E-mail: tianjin_tcm001@sina.com

*Contributed equally

Key words: absorption, buccal mucosa absorption, Su Xiao Jiu Xin dripping pill, cardiovascular and cerebrovascular disease and $n$-butylphthalide) and organic acids (ferulic acid) from Rhizoma Chuanxiong $(2,3)$. Pharmacological studies have also revealed that major active components, including borneol, isoborneol, ligustilide, $n$-butylphthalide and ferulic acid, are implicated in the treatment of cardiovascular and cerebrovascular diseases (4-7). Therefore, an efficient method should be developed to detect these compounds absorbed in the buccal mucosa.

SXJXDP is sublingually administered and can be absorbed by the rich blood flow in the buccal mucosa. As a non-keratinising epithelial tissue, the buccal mucosa provides a number of advantages in drug permeability, prevents first-pass metabolism in the liver exhibiting good compliance depending on a particular drug and contains efficient enzymatic flora $(8,9)$. Therefore, the characteristic of drug absorption in the human buccal mucosa should be determined to provide relevant guidelines. However, to the best of our knowledge, the release pattern and absorption characteristics of the main bioactive components of SXJXDP in the human buccal mucosa have not yet been reported.

In the present study, borneol, isoborneol, $n$-butylphthalide and ligustilide were detected by gas chromatography-mass spectrometry (GC-MS). Ferulic acid was analysed by high-performance liquid chromatography (HPLC). The circulating system involved in the drug absorption in the buccal mucosa was observed to investigate the absorption characteristics of the five main bioactive components, which are associated with cardiovascular and cerebrovascular diseases. Furthermore, an absorption curve was illustrated and permeability coefficient was calculated. The study aimed to establish efficient methods and explain the absorption characteristics of SXJXDP.

\section{Materials and methods}

Materials. The standard compounds of borneol, isoborneol, ligustilide, $n$-butylphthalide and ferulic acid were purchased from the National Institutes for Food and Drug Control (Beijing, China). HPLC-grade acetonitrile, $n$-hexane and dichloromethane were purchased from Oceanpak Alexative Chemical (Gothenburg, Sweden). Pure water was purchased 
from Wahaha Group Co. (Hangzhou, China). SXJXDP was purchased from the No. 6 Pharmaceutical Factory of Tianjin Zhongxin Pharmaceutical Co. (Tianjin, China). Six healthy volunteers underwent training and signed an informed consent prior to the experiment. Our study was approved by the ethics comitee of Tanjin University of Traditional Chinese Medicine. These volunteers gargled pure water prior to the experiment. The presence or absence of adverse reactions was closely observed during the process and whether the mucosa remains normal or becomes abnormal following the completion of the experiment was assessed.

In total, 15 pills were dissolved in $50 \mathrm{ml}$ purified water and added to the circulating fluid storage cup of the circulating devices. The buccal mucosa devices used in the experiment were prepared in accordance with previously described methods $(10,11)$. Fig. 1 illustrates the diffusion cell used in the study. This diffusion cell is composed of polytetrafluoroethylene and comprises two sides; a-side functions as an inlet of circulating fluid and b-side serves as an outlet of circulating fluid $(10,11)$. The circulating system was equipped with a magnetic stirrer and performed under the following conditions: A suitable modulation speed and solution temperature of $37^{\circ} \mathrm{C}$. At $0,1,3,5,10,20,30,45,60$ and $90 \mathrm{~min}, 300 \mu 1$ of the solution was obtained. Subsequent to performing the sampling, the same volume of blank solution was immediately injected as a supplementary sample.

Chromatography. Borneol, isoborneol, ligustilide and $n$-butylphthalide were analysed using a GC-MS-QP-2010 SE (Shimadzu, Japan) with a column Rtx-5MS type at an inlet temperature of $280^{\circ} \mathrm{C}$, an ion source temperature of $200^{\circ} \mathrm{C}$, a flow rate of $1 \mathrm{ml} / \mathrm{min}$ helium carrier gas and an injection volume of $1 \mu \mathrm{l}$ in a splitless mode.

Borneol and isoborneol samples were prepared under the following chromatographic conditions. In brief, $50 \mu 1$ sample was obtained and $300 \mu \mathrm{l} n$-hexane were added. Subsequently, dichloromethane $(9: 1 \mathrm{v} / \mathrm{v})$ liquid-to-liquid extraction was performed by vortexing for $1 \mathrm{~min}$, and the vortexed extract was centrifuged at $9,168 \times \mathrm{g}$ for $5 \mathrm{~min}$. An aliquot of the supernatant was subsequently obtained. The temperature program started at $70^{\circ} \mathrm{C}$ for $2 \mathrm{~min}$ and was increased at a rate of $5^{\circ} \mathrm{C} / \mathrm{min}$ until $120^{\circ} \mathrm{C}$ was reached. Temperature was ramped at $50^{\circ} \mathrm{C} / \mathrm{min}$ until a final temperature of $280^{\circ} \mathrm{C}$ was achieved. Temperature was maintained constantly at $280^{\circ} \mathrm{C}$ for $2 \mathrm{~min}$.

Ligustilide and $n$-butylphthalide samples were prepared under the following chromatographic conditions. In brief, $100 \mu 1$ sample was obtained and $100 \mu 1 n$-hexane was added. Subsequently, dichloromethane $(9: 1 \mathrm{v} / \mathrm{v})$ liquid-to-liquid extraction was performed by vortexing for $1 \mathrm{~min}$. The vortexed extract was centrifuged at 9,168 x $\mathrm{g}$ for $5 \mathrm{~min}$. An aliquot of the supernatant was subsequently obtained. The temperature program started at $70^{\circ} \mathrm{C}$ for $2 \mathrm{~min}$ and was increased at a rate of $5^{\circ} \mathrm{C} / \mathrm{min}$ until $120^{\circ} \mathrm{C}$ was reached. Temperature was ramped at $50^{\circ} \mathrm{C} / \mathrm{min}$ until a final temperature of $300^{\circ} \mathrm{C}$ was reached, and was subsequently maintained constantly at $300^{\circ} \mathrm{C}$ for 2 min.

Ferulic acid sample was prepared under the following chromatographic conditions. In brief, $100 \mu \mathrm{l}$ sample was filtered using a $0.45 \mu \mathrm{m}$ membrane and injected into HPLC-LC-20AT (Shimadzu, Japan). HPLC was performed in a Diamonsil

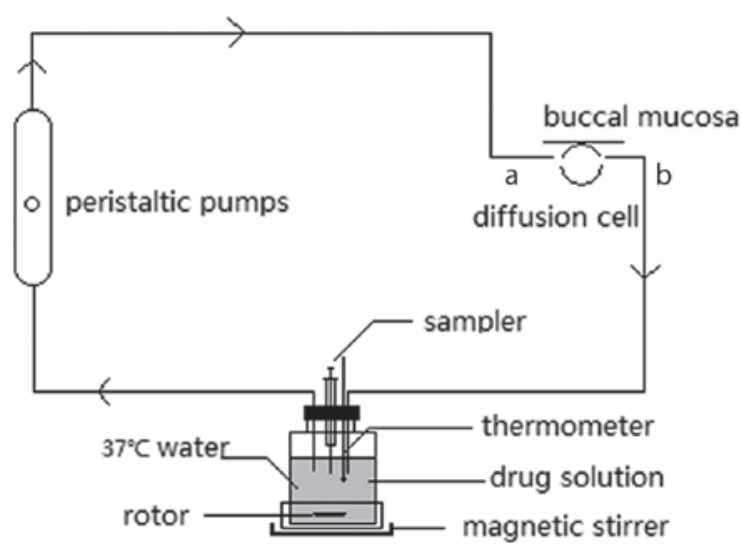

Figure 1. Design of the buccal mucosa devices. a, inlet of circulating fluid; $b$, outlet of circulating fluid.

C18 column $(4.6 \times 250 \mathrm{~mm}, 5 \mu \mathrm{m})$ with a mobile phase of methanol: $1 \%$ acetic acid aqueous solution $(42: 58 \mathrm{v} / \mathrm{v})$ at a flow rate of $1 \mathrm{ml} / \mathrm{min}$. The column temperature was maintained at $25^{\circ} \mathrm{C}$ and the injection volume was $20 \mu 1$. The wavelength of the detector was set at $321 \mathrm{~nm}$.

The cumulative absorption rate was calculated to evaluate drug absorption and was obtained using the following equation: $\left(F_{0}-F_{t}\right) / F_{0} \times 100 \%$, where $F_{t}$ is the liquid concentration at time $t$ and $F_{0}$ is the initial concentration of the solution.

The permeability coefficient was calculated to evaluate drug permeability. Permeability coefficient was obtained using the following equations: $J=\mathrm{d} Q / \mathrm{d} t=P \times\left(C_{d}-C_{r}\right) \approx P \times C_{d}$, $P=J / C_{d} \times 60$, where $Q$ is the drug cumulative infiltration capacity in $\mu \mathrm{g} / \mathrm{cm}^{2} ; t$ is the time in min; $P$ is the permeability coefficient in $\mathrm{cm}^{2} / \mathrm{s}$; and $C_{d}$ and $C_{r}$ are the drug concentrations of the supply cell and the accepting cell in $\mu \mathrm{g} / \mathrm{ml}$, respectively. Additionally, $C_{d} \gg C_{r}$.

Under these conditions, three sensitive and reliable analytical methods were developed to simultaneously quantify borneol, isoborneol, ligustilide and $n$-butylphthalide by GC-MS and analyse ferulic acid by HPLC. The representative chromatograms of the compounds indicated that the developed method was free of any evident interference.

\section{Results and Discussion}

GC-MS and HPLC methods were validated in terms of linearity, precision, accuracy, stability and recovery. Calibration curves were constructed at five different concentration levels from the following concentration ranges: $2-200 \mu \mathrm{g} / \mathrm{ml}$ for borneol; $2-400 \mu \mathrm{g} / \mathrm{ml}$ for isoborneol; $1-100 \mu \mathrm{l} / \mathrm{ml}$ for ligustilide; $0.1-15 \mu \mathrm{l} / \mathrm{ml}$ for $n$-butylphthalide; and 1.081-16.215 $\mu \mathrm{g} / \mathrm{ml}$ for ferulic acid. Each calibration curve was obtained from five different concentrations of the five bioactive analytes by linear regression analysis. The obtained correlation coefficient values $(r>0.999)$ indicated good correlations between the concentrations and their peak responses within the test ranges. Stability test results showed that the processed samples could be re-injected after $0,2,4,8$ and $12 \mathrm{~h}$; furthermore, the relative standard deviation (RSD)\% of the stabilities of these five bioactive analytes ranged, $1.11-8.71 \%$, which was within acceptable ranges. The precision of these methods was determined by 
Table I. Result of the method validation.

\begin{tabular}{|c|c|c|c|c|c|c|c|c|}
\hline \multirow[b]{2}{*}{ Compound } & \multicolumn{2}{|c|}{ Calibration curve } & \multirow{2}{*}{$\begin{array}{c}\text { Precision, } \\
\text { RSD\% }\end{array}$} & \multirow{2}{*}{$\begin{array}{c}\text { Accuracy, } \\
\text { RSD } \%\end{array}$} & \multirow{2}{*}{$\begin{array}{c}\text { Stability, } \\
\text { RSD\% }\end{array}$} & \multicolumn{3}{|c|}{ Recovery } \\
\hline & Regression equation & $\mathrm{r}$ & & & & Low & Med & High \\
\hline Borneol & $y=1.311 \times 10^{5} x-3.329 \times 10^{4}$ & 0.9998 & 3.58 & 2.44 & 2.63 & 88.96 & 91.63 & 90.61 \\
\hline Isoborneol & $y=4.148 \times 10^{4} x+3.203 \times 10^{5}$ & 0.9994 & 4.54 & 2.95 & 2.46 & 86.80 & 87.84 & 86.27 \\
\hline Ligustilide & $y=2.594 \times 10^{4} x-2.317 \times 10^{4}$ & 0.9998 & 5.01 & 4.86 & 8.71 & 88.77 & 89.25 & 84.67 \\
\hline$n$-butylphthalide & $y=1.631 \times 10^{5} x-8.477 \times 10^{3}$ & 0.9996 & 2.73 & 8.71 & 3.86 & 87.50 & 88.99 & 84.72 \\
\hline Ferulic acid & $y=1.112 \times 10^{5} x-3.830 \times 10^{3}$ & 0.9998 & 1.12 & 1.85 & 1.11 & 103.77 & 100.32 & 100.07 \\
\hline
\end{tabular}

RSD, relative standard deviation.

repeated injections $(n=6)$ of the standard solutions; the accuracy of these methods was determined by injections $(n=6)$ of equally treated samples. The $\mathrm{RSD} \%$ values of the precision and the accuracy of five bioactive analytes ranged, 1.12-5.01\% and $1.85-8.71 \%$, respectively. The recoveries of the GC-MS analytes were compared to a standard solution following extraction using known concentrations at three different levels. The recoveries of the HPLC analytes were determined by spiking the samples with known amounts of the standard solution at three different concentration levels. The recoveries of the five analytes were as follows: $88.96-91.63 \%$ for borneol; $86.27-87.84 \%$ for isoborneol; $84.67-89.25 \%$ for ligustilide; $84.72-88.99 \%$ for $n$-butylphthalide; and $100.07-103.77 \%$ for ferulic acid. Overall, the recoveries of each of the analytes at three different concentrations were consistent and $>80 \%$. These results demonstrated that all the values were present in permissible ranges and the methods were accurate (Table I).

The buccal mucosa absorption curve showed that the five types of components reached a plateau in 10-20 min. During this period, the maximum cumulative absorption rate was almost obtained. Subsequently, the cumulative absorption rate was significantly decreased. The cumulative absorption rates of borneol, isoborneol, ligustilide and $n$-butylphthalide showed that the maximum accumulation in $60 \mathrm{~min}$ were 86.70 , 93.16, 94.25 and $93.16 \%$, respectively.

The accumulation of the four components was almost complete. In the buccal mucosa absorption curve of ferulic acid in $60 \mathrm{~min}$, the maximum absorption rate was $15.91 \%$ with incomplete absorption and each component exhibited different performances (Fig. 2).

Ferulic acid yielded the lowest permeability coefficient; by comparison, the four other components exhibited relatively similar permeability coefficients. However, the penetration ability of ferulic acid in the buccal mucosa was $~ 10$ times weaker than that of the four other components (Table II).

Previous studies showed that SXJXDPs are stable during absorption (1). This phenomenon can account for the decrease in drug concentration in vitro, indicating that this decrease is not caused by drug degradation; instead, this decrease occurs as a result of drug absorption into the bloodstream. Therefore, residual drug method was applied in the present study to evaluate absorption.

Components undergo rapid sublingual absorption as the buccal mucosa is extremely thin and rich in blood vessels.
Table II. Permeability coefficient of buccal mucosa.

\begin{tabular}{lc}
\hline Compound & $\mathrm{P}, \mathrm{cm} / \mathrm{s}$ \\
\hline Borneol & $1.12 \times 10^{-2}$ \\
Isoborneol & $1.17 \times 10^{-2}$ \\
Ligustilide & $1.45 \times 10^{-2}$ \\
$n$-butylphthalide & $1.34 \times 10^{-2}$ \\
Ferulic acid & $1.93 \times 10^{-3}$ \\
\hline
\end{tabular}

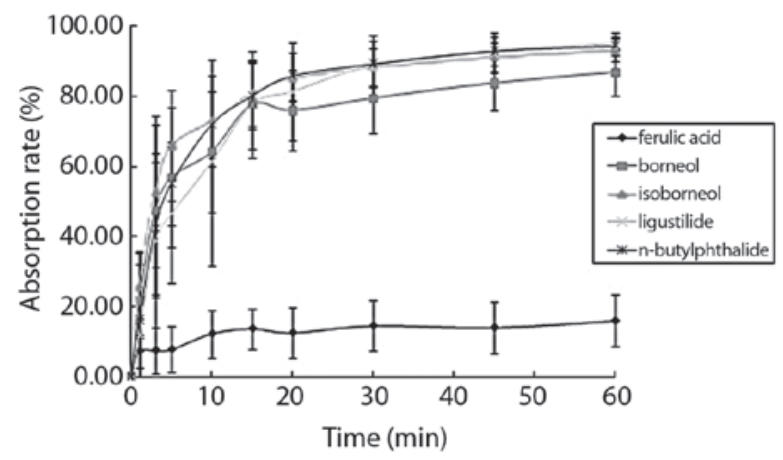

Figure 2. Time course of the cumulative absorption rates of the five components of Su Xiao Jiu Xin dripping pill. Values are mean \pm standard deviation.

These components can enter blood circulation within an extremely short time to alleviate symptoms and provide first-aid relief. Drug absorption occurs in two passages, via intracellular and intercellular routes, which primarily exhibit passive diffusion influenced by several physicochemical properties, such as lipid solubility, dissociation degree, molecular weight and permeability effect.

In general, drugs with low molecular weights are easily absorbed by mucosa capillaries. The five main bioactive components exhibit molecular weights $<200 \mathrm{Da}$; as such, these components can pass through the buccal mucosa membrane and into the bloodstream. As a drug-promoting agent in TCM, borneol exhibits strong absorption and permeation abilities, and can also promote the absorption of other substances in the buccal mucosa to a certain extent (12).

Lipophilic drugs can easily penetrate intercellular channels filled with lipid. This penetration is possible as sublingually 
administered drugs permeability coefficients closely associated with the oil-water partition, $K$. $K$ is extremely high when fat-soluble compounds are insoluble in saliva. By contrast, $K$ is extremely low when compounds are strongly hydrophilic and membrane permeability is weak. The Ideal $K$ ranges, 40-2,000 (13,14). Borneol, isoborneol, $n$-butylphthalide and ligustilide are fat-soluble compounds; therefore, these compounds are absorbed more efficiently and rapidly in the buccal mucosa compared to ferulic acid. In contrast to the four fat-soluble compounds, ferulic acid exhibits strong hydrophilicity, poor permeation ability, poor absorption and weak permeability in the buccal mucosa. In addition, the pKa values of ferulic acid are 4.56 and 8.65; the main form of ferulic acid in saliva ( $\mathrm{pH}=6.6-7.1)(15)$ is a mono-anion (16). Drugs are transported across the cell membrane in an absorbable molecular state. However, the present form of ferulic acid is one of the key reasons of its low absorption. Therefore, drug absorption in the buccal mucosa is an extremely complicated process.

In conclusion, the GC-MS and HPLC methods were established in the present study to detect the bioactive components of SXJXDP. These methods may be suitably applied to elucidate the characteristics and permeabilities of drugs in the buccal mucosa of healthy volunteers, as validated by selectivity, linearity, precision and recovery test results. Novel efficient methods should be developed to implement comprehensive quality control strategies of drugs absorbed in the buccal mucosa.

\section{Acknowledgements}

The present study was financially supported by the Large Variety of Technological Innovation of Su Xiao Jiu Xin Dripping Pill (grant nos. 2011ZX09201-201 and 2011ZX09201-201-2).

\section{References}

1. Sun SR, Huang X and Zhang L: Advances in studies on pharmacokinetics, pharmacodynamics and clinical research of Su Xiao Jiu Xin Wan. Chin Tradit Herb Drugs 33: 89-91, 2002.
2. Wei Y,Huang W and Gu Y: Online isolation and purification of four phthalide compounds from Chuanxiong rhizoma using high-speed counter-current chromatography coupled with semi-preparative liquid chromatography. J Chromatogr A 1284: 53-58, 2013.

3. Alaerts G, Merino-Arevalo M, Dumarey M, et al: Exploratory analysis of chromatographic fingerprints to distinguish rhizoma Chuanxiong and rhizoma Ligustici. J Chromatogr A 1217: 7706-7716, 2010

4. Xiang L, Jiang P, Zhan C, et al: The serum metabolomic study of intervention effects of the traditional Chinese medicine Shexiang Baoxin Pill and a multi-component medicine polypill in the treatment of myocardial infarction in rats. Mol BioSyst 8: 2434-2442, 2012.

5. Diao X, Deng P, Xie C et al: Metabolism and pharmacokinetics of 3-n-butylphthalide (NBP) in humans: the role of cytochrome P450s and alcohol dehydrogenase in biotransformation. Drug Metab Dispos 41: 430-444, 2013.

6. Feng Z, Lu Y, Wu X, et al: Ligustilide alleviates brain damage and improves cognitive function in rats of chronic cerebral hypoperfusion. J Ethnopharmacol 144: 313-321, 2012.

7. Alam MA, Sernia C and Brown L: Ferulic acid improves cardiovascular and kidney structure and function in hypertensive rats. J Cardiovasc Pharmacol 61: 240-249, 2013.

8. Patel VF, Liu F and Brown MB: Modeling the oral cavity: in vitro and in vivo evaluations of buccal drug delivery systems. J Control Release 161: 746-756, 2012.

9. Campisi G, Paderni C, Saccone R, Di Fede O, Wolff A and Giannola LI: Human buccal mucosa as an innovative site of drug delivery. Curr Pharm Des 16: 641-652, 2010.

10. Adrian CL, Olin HB, Dalhoff K and Jacobsen J: In vivo human buccal permeability of nicotine. Int J Pharm 311: 196-202, 2006.

11. Zhang X, Wang L, Yang X, et al: Study on the correlation between buccal absorption in healthy volunteers and release rate in vitro of Glycyrrhizin in Qingyan Drop Pills. Chin Med J Res Prac 24: 60-61, 2010.

12. Senel S and Hincal AA: Drug permeation enhancement via buccal route: possibilities and limitations. J Control Release 72: 133-144, 2001.

13. Yuji $\mathrm{K}$ and Toshikiro K: Drug absorption by oral mucosa-mechanism of absorption and promoter. Int J Clin Exp Med 145-147: 468-470, 1988.

14. Zhou Y, Li W, Chen L, Ma S, Ping L and Yang Z: Enhancement of intestinal absorption of akebia saponin D by borneol and probenecid in situ and in vitro. Environ Toxicol Pharmacol 29: 229-234, 2010

15. Leon-Carmona JR, Alvarez-Idaboy JR and Galano A: On the peroxyl scavenging activity of hydroxycinnamic acid derivatives: mechanisms, kinetics, and importance of the acid-base equilibrium. Phys Chem Chem Phys 14: 12534-12543, 2012.

16. Bikov A, Galffy G, Tamasi L, Lazar Z, Losonczy G and Horvath I: Exhaled breath condensate $\mathrm{pH}$ is influenced by respiratory droplet dilution. J Breath Res 6: 046002, 2012. 